\title{
Heisenberg limit for displacements with semiclassical states
}

\author{
Alfredo Luis* \\ Departamento de Óptica, Facultad de Ciencias Físicas, Universidad Complutense, 28040 Madrid, Spain
}

(Received 27 July 2003; published 1 April 2004)

\begin{abstract}
We analyze the quantum limit to the sensitivity of the detection of small displacements. We focus on the case of free particles and harmonic oscillators as the systems experiencing the displacement. We show that the minimum displacement detectable is proportional to the inverse of the square root of the mean value of the energy in the state experiencing the displacement (Heisenberg limit). We present a measuring scheme that reaches this limit using semiclassical states. We examine the performance of this strategy under realistic practical conditions by computing the effect of imperfections such as losses and nonunit detection efficiencies. This analysis confirms the robustness of this measuring strategy by showing that the experimental imperfections can be suitably compensated by increasing the mean energy of the input state.
\end{abstract}

DOI: 10.1103/PhysRevA.69.044101

PACS number(s): 42.50.Lc, 03.65.Sq, 42.50.St

\section{INTRODUCTION}

The continual improvement of the experimental techniques entails that the issue of quantum fluctuations is currently a matter of practical interest. This is the case of the limits that quantum fluctuations place on the sensitivity of the detection of small signals. In general, these limits depend on the energy resources, that is to say, on the number of particles (usually photons) involved in the measurement. For example, the quantum limit to optical phase-shift detection is expressed by the so-called Heisenberg limit: the minimum detectable phase shift is inversely proportional to the energy or the number of photons employed [1,2]. We show below that a similar result applies to the detection of small displacements: the minimum detectable displacement is inversely proportional to the square root of the mean value of the energy. We prove this for free particles and harmonic oscillators, which cover all the cases of interest in this context.

Most approaches to the problem of quantum limits conclude that the optimum arrangements reaching the ultimate accuracy unavoidably require the use of nonclassical states, usually squeezed or number states [2]. However, nonclassical states are extremely fragile and their use imposes very restrictive experimental conditions in order to protect them against practical imperfections, such as losses and inefficient detectors, that seriously degrade their performance.

In a previous work [3] it has been shown that, contrary to common belief, the measurement of phase shifts can approach the Heisenberg limit without using nonclassical states. In this work we extend this idea to the measurement of small displacements showing that the Heisenberg limit can be reached by using exclusively semiclassical coherent states. Moreover, we examine the performance of this strategy under realistic practical conditions by computing the effect of experimental imperfections, such as losses and nonunit detection efficiencies. Finally, we compare the performance of this approach with the behavior of more standard arrangements that reach the Heisenberg limit using nonclassical squeezed states.

\footnotetext{
*Electronic address: alluis@ fis.ucm.es
}

\section{HEISENBERG LIMIT FOR DISPLACEMENTS}

In this section we examine the limits imposed by quantum mechanics to the detection of small displacements. We consider a system described by two conjugate, unbounded, adimensional, Cartesian variables represented by the operators $X, P$, with commutator $[X, P]=i$. The corresponding annihilation operator is $a=(X+i P) / \sqrt{2}$. These variables can represent position and linear momentum for a material particle, or the field quadratures of an electromagnetic field mode. For the sake of simplicity the units have been chosen so that all variables are adimensional and masses, frequencies, and $\hbar$ are unity.

The system is prepared in a known input state $|\psi\rangle$ which experiences a small displacement transforming $X$ into $X$ $\rightarrow X+\lambda$. The objective is to infer $\lambda$ with the minimum error from a measurement performed on the displaced state

$$
\left|\psi^{\prime}\right\rangle=e^{-i \lambda P}|\psi\rangle
$$

In a first approach to the problem, we might consider the natural choice of $X$ as the measured observable. In such a case the uncertainty $\Delta \lambda$ of the inferred value will be given by the uncertainty of $X$ in the state $|\psi\rangle, \Delta \lambda=\Delta X$ (see Fig. 1). If the initial state is a semiclassical coherent state $|\alpha\rangle$, with $a|\alpha\rangle=\alpha|\alpha\rangle$, we have $\Delta \lambda=\Delta X=1 / \sqrt{2}$, irrespective of $\alpha$.

The above resolution can be improved if we resort to the nonclassical squeezed states

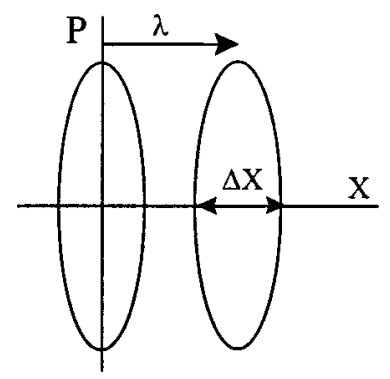

FIG. 1. Illustration of the resolution achievable in the detection of a small displacement $\lambda$. The ellipses represent the phase-space area enclosed by the state $|\psi\rangle$. 


$$
|\xi\rangle=e^{r\left(a^{2}-a^{\dagger 2}\right) / 2}|0\rangle,
$$

where $|0\rangle$ is the vacuum state, $a|0\rangle=0$. These states present reduced quantum fluctuations below the vacuum level,

$$
\Delta X=\frac{1}{\sqrt{2}} e^{-r},
$$

improving by a factor $e^{-r}$ the resolution achievable with coherent states. Some other proposals to improve the precision by using entangled states and Schrödinger cat states can be found in Refs. [4,5].

Our objective in this section is to demonstrate that, for arbitrary $|\psi\rangle$, the minimum value for $\Delta X$ is limited by the mean value of the energy $\bar{H}=\langle\psi|H| \psi\rangle$ conveyed by $|\psi\rangle$, where $H$ represents the Hamiltonian of the system. For definiteness, we consider the two most relevant situations: the free particle and the harmonic oscillator. For the free particle we have

$$
\bar{H}=\frac{1}{2} \bar{P}^{2}=\frac{1}{2}\left[(\Delta P)^{2}+\bar{P}^{2}\right] \geqslant \frac{1}{2}(\Delta P)^{2} \geqslant \frac{1}{8(\Delta X)^{2}},
$$

where we have used the uncertainty relation $\Delta X \Delta P \geqslant 1 / 2$. On the other hand, for the harmonic oscillator

$$
\begin{aligned}
\bar{H}= & \frac{1}{2}\left(\bar{P}^{2}+\bar{X}^{2}\right)=\frac{1}{2}\left[(\Delta P)^{2}+(\Delta X)^{2}+\bar{P}^{2}+\bar{X}^{2}\right] \\
& \geqslant \frac{1}{2}\left[\frac{1}{4(\Delta X)^{2}}+(\Delta X)^{2}\right] \geqslant \frac{1}{8(\Delta X)^{2}},
\end{aligned}
$$

where in the last inequality we have considered that in our case $\Delta X \ll 1$.

The conclusion we obtain is that the free particle and the harmonic oscillator lead to the same relation between uncertainty and energy,

$$
\Delta \lambda=\Delta X \geqslant \frac{1}{\sqrt{8 \bar{H}}} .
$$

For the free particle the equality is reached by minimum uncertainty states with $\bar{P}=0$. For the harmonic oscillator the equality is approached by minimum uncertainty states with $\bar{P}=\bar{X}=0$, provided that $\Delta X \ll 1$. For example, this is the case of the squeezed states (2.2) when $r \gg 1$.

We can see that the uncertainty is proportional to the inverse of the square root of the mean energy (or mean number of photons for a single-mode electromagnetic field). We have obtained also that the nonclassical character of the state experiencing the displacement (squeezed states) is necessary in order to reach the maximum resolution with this measuring strategy.

We can refer to Eq. (2.6) as the Heisenberg limit for small displacements. This agrees well with the concept of Heisenberg limit for phase shifts. This is because when $\bar{X}=0$ a small displacement $\lambda$ can be related to a small phase shift $\phi$ $\simeq \lambda / \bar{P}$ as illustrated in Fig. 2. Since in general $\bar{P} \leqslant \sqrt{2 \bar{H}}$ we

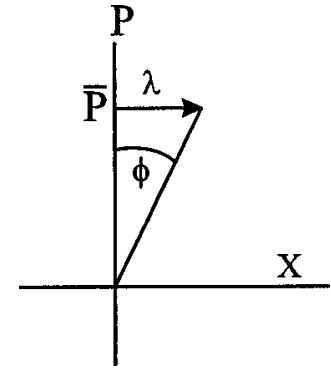

FIG. 2. Illustration of the relation between a small displacement $\lambda$ and a small phase shift $\phi$.

have $\Delta \phi \simeq \Delta \lambda / \bar{P} \geqslant 1 /(4 \bar{H})$, which is the standard form for the Heisenberg limit for phase-shift measurements.

It is worth noting that in all the approaches to the problem the Heisenberg limit refers exclusively to the energy conveyed by the state undergoing the shift (the probe) and does not take into account other energy resources (if any) that might be involved in the preparation of the input state (the generation of squeezed states in nonlinear crystals requires intense pump fields) or in the realization of the measurement (position measurements may require illumination, and quadrature measurements require intense local oscillators). To some extent, it is at the probe stage where quantum complementarity is clearly at work leading to a precisionenergy balance of quantum origin. The preparation and measuring stages cannot improve the precision beyond the limit imposed by the quantum nature of the probe, which scales as dictated by the Heisenberg limit.

Finally let us note that there is another quantum limit in the context of precise detection of small forces, the so-called standard quantum limit, that applies to the detection of displacements occurring between two or more consecutive measurements [6].

\section{HEISENBERG LIMIT WITH SEMICLASSICAL STATES}

In this section we show that it is possible to reach the Heisenberg limit in the detection of small displacements without using nonclassical states. To this end let us consider the following generator of displacements $G=|+\rangle\langle+| P$ so that $X$ is transformed as $X \rightarrow X+\lambda|+\rangle\langle+|$, where $| \pm\rangle$ denote two orthogonal states of an auxiliary system. For the sake of definiteness, we focus on the case of a single electromagnetic field mode coupled with an effective two-level atom with internal energy levels $| \pm\rangle$.

The initial state of the whole system factorizes as

$$
|\Psi\rangle=|\varphi\rangle|\alpha\rangle,
$$

where

$$
|\varphi\rangle=\frac{1}{\sqrt{2}}(|-\rangle+i|+\rangle),
$$

and $|\alpha\rangle$ denotes again a coherent state. After the displacement, the state is 


$$
\left|\Psi^{\prime}\right\rangle=e^{-i \lambda G}|\Psi\rangle=\frac{1}{\sqrt{2}}\left(|-\rangle|\alpha\rangle+i e^{i \theta}|+\rangle|\alpha+\lambda / \sqrt{2}\rangle\right),
$$

where $\theta=\lambda i\left(\alpha-\alpha^{*}\right) /(2 \sqrt{2})$. In order to infer $\lambda$ we consider a measurement performed on the auxiliary system described by projection on the states,

$$
\left|\varphi_{ \pm}\right\rangle=\frac{1}{\sqrt{2}}(|-\rangle \pm|+\rangle)
$$

There are only two possible outcomes $(+$ and -$)$ that appear with probabilities

$$
\begin{aligned}
p_{ \pm} & =\frac{1}{2}\left[1 \pm \operatorname{Re}\left(i e^{i \theta}\langle\alpha \mid \alpha+\lambda / \sqrt{2}\rangle\right)\right] \\
& =\frac{1}{2}\left\{1 \pm \operatorname{Re}\left[i e^{\lambda\left(\alpha^{*}-\alpha\right) / \sqrt{2}} e^{-\lambda^{2} / 4}\right]\right\} .
\end{aligned}
$$

Optimum results are obtained when $\alpha=-\alpha^{*}=i \sqrt{\bar{n}}$, where $\bar{n}$ represents the mean number of photons. Since we are interested in the case $\lambda \ll 1$ we get

$$
p_{ \pm}=\frac{1}{2}[1 \pm \sin (\lambda \sqrt{2 \bar{n}})] .
$$

The condition $\lambda \ll 1$ is the only prior information about the signal assumed in this work. Actually this is not a restrictive condition since this is the case in most practical situations in the context of precision measurements. The fulfillment of this assumption can be based on theoretical considerations (an upper bound for the expected signal is enough) or even via a prior rough measurement (for example, this is the case of precision spectroscopy where several observation schemes can be arranged in a series of increasing resolution).

In order to obtain meaningful conclusions the measurement must be repeated several times. After $N$ repetitions, the probability that the outcome + is obtained $m$ times is given by the binomial distribution,

$$
P_{N}(m)=\left(\begin{array}{l}
N \\
m
\end{array}\right) p_{+}^{m} p_{-}^{N-m}
$$

In the limit of large $N$ the quotient $m / N$ can be regarded as effectively continuous and the binomial distribution tends to be Gaussian,

$$
P(\tilde{\lambda}) \simeq \sqrt{\frac{N \bar{n}}{\pi}} e^{-N \bar{n}(\tilde{\lambda}-\lambda)^{2}}
$$

where $\tilde{\lambda}=(2 m-N) /(N \sqrt{2 \bar{n}})$, so that the data analysis becomes very simple. We can appreciate that $\tilde{\lambda}$ is a suitable estimator of the true but unknown $\lambda$. The uncertainty of this estimation is

$$
\Delta \tilde{\lambda}=\frac{1}{\sqrt{2 \bar{n} N}} .
$$

Therefore, this scheme reaches the Heisenberg limit since $\bar{n} N$ represents the total number of particles used in the measurement.
Concerning the practical realization of the generator $G$ $=|+\rangle\langle+| P$ we can refer to a nonresonant atom-field interaction governed by a Hamiltonian of the form $H_{\text {int }} \propto|+\rangle$ $\times\langle+| A^{\dagger} A$ [7], where $A \propto a+b$. If the auxiliary mode $b$ is in a semiclassical state of large amplitude we can safely replace $b$ by a classical complex amplitude $b \simeq \beta$. The final form of the generator is obtained retaining the leading terms in powers of $\beta$.

\section{NOISE ANALYSIS}

The above analysis assumes ideal conditions. Next we show that the method presented in this work is robust against practical imperfections. This can be demonstrated by examining the uncertainty after including standard imperfections, such as losses and nonunit detector efficiencies. The analysis of losses is greatly simplified by the fact that the field state is coherent and we are always considering a single-mode approach. Then, the state remains always coherent with a decreased amplitude $|\sqrt{\eta} \alpha\rangle$, where $1-\eta$ is the percentage of lost photons [8].

On the other hand, in a first simple analysis, the atomic losses and inefficient atomic detection can be taken into account simultaneously by replacing the pure state $|\varphi\rangle$ by the mixed state,

$$
|\varphi\rangle\langle\varphi|\rightarrow p| \varphi\rangle\langle\varphi|+\frac{1-p}{2} I
$$

where $I=|+\rangle\langle+|+|-\rangle\langle-|$ is the identity and $p$ represents the fraction of atoms remaining in the prepared state (3.2).

These sources of error affect the statistics of the measurement leading to the replacement $p_{ \pm} \rightarrow p_{ \pm}^{\prime}$, where

$$
p_{ \pm}^{\prime}=p p_{ \pm}+\frac{1}{2}(1-p) \simeq \frac{1}{2}[1 \pm p \sin (\lambda \sqrt{2 \eta \bar{n}})] .
$$

The same Gaussian approximation leading from the binomial (3.7) to the Gaussian (3.8) leads in this case a phase uncertainty of the form

$$
\Delta \lambda=\frac{\Delta \lambda_{\text {ideal }}}{p \sqrt{\eta}}=\frac{1}{p \sqrt{2 \eta \bar{n} N}} .
$$

The main conclusion is that the degradation of the measurement is not critical, since it can be suitably compensated by a proper increase of the number of photons and atoms.

On the other hand, we can carry out a similar analysis for the arrangement based on the measurement of $X$ when the system is prepared in a nonclassical squeezed state $|\xi\rangle$. In such a case the joint effect of losses and nonideal detectors can be computed as the effect of a fictitious beam splitter of transmittance $\eta$ placed in front of an ideal detector [9]. This leads to a quadrature uncertainty $\Delta X$ of the form

$$
(\Delta X)^{2}=\eta(\Delta X)_{\text {ideal }}^{2}+\frac{1}{2}(1-\eta),
$$

where $\Delta X_{\text {ideal }}$ represents the uncertainty (2.3). In this case, the practical errors cannot be compensated by increasing the mean number of photons. For example, for finite $\eta$ and $r \gg 1$ we have $(\Delta X)^{2} \rightarrow(1-\eta) / 2$ that no longer depends on the number of photons. 
In other words, the key point for the robustness of the scheme analyzed in this work is that the field is always in a semiclassical coherent state, since for $\lambda \ll 1$ we have $\left|\left\langle\alpha\left|e^{-i \lambda P}\right| \alpha\right\rangle\right| \simeq 1$. Moreover, no entanglement is produced during the process. This is in spite of the fact that the above Hamiltonian $G$ can be used to create highly exotic nonclassical states, as demonstrated in Ref. [10]. In our case the role of $G$ is to transfer the signal from the field to the auxiliary system, instead of producing nonclassical states.

\section{CONCLUSIONS}

We have presented a robust measuring strategy for the detection of small displacements that reaches the quantum limit (Heisenberg limit) using exclusively semiclassical coherent states. The semiclassical character of the state experiencing the displacement is essential for the robustness of the method against practical imperfections. This allows us to use states with a large number of particles (leading to an accordingly large resolution) without experiencing the degradation of the performance suffered by the schemes reaching the Heisenberg limit using nonclassical states.

\section{ACKNOWLEDGMENT}

This work was supported by Project No. PR1/03-11630 of the University Complutense.
[1] Z. Y. Ou, Phys. Rev. A 55, 2598 (1997).

[2] A. Luis and L. L. Sánchez-Soto, Progress in Optics, edited by E. Wolf (Elsevier, Amsterdam, 2000), Vol. 41, p. 421.

[3] A. Luis, Phys. Rev. A 65, 025802 (2002).

[4] G. M. D'Ariano and M. F. Sacchi, Phys. Rev. A 52, R4309 (1995).

[5] W. J. Munro, K. Nemoto, G. J. Milburn, and S. L. Braunstein, Phys. Rev. A 66, 023819 (2002).

[6] C. M. Caves, K. S. Thorne, R. W. P. Drever, V. D. Sandberg, and M. Zimmermann, Rev. Mod. Phys. 52, 341 (1980); H. P. Yuen, Phys. Rev. Lett. 51, 719 (1983); M. H. Partovi and R. Blankenbecler, ibid. 57, 2891 (1986); M. Ozawa, ibid. 60,
385 (1988).

[7] M. Brune, S. Haroche, V. Lefevre, J. M. Raimond, and N. Zagury, Phys. Rev. Lett. 65, 976 (1990); H. Paul, Quantum Opt. 3, 169 (1991).

[8] M. O. Scully and M. S. Zubairy, Quantum Optics (Cambridge University Press, Cambridge, 1997).

[9] H. P. Yuen and J. H. Saphiro, IEEE Trans. Inf. Theory IT-26, 78 (1980); B. Yurke, Phys. Rev. A 32, 311 (1985); T. Kim, Y. Ha, J. Shin, H. Kim, G. Park, K. Kim, T.-G. Noh, and Ch. K. Hong, ibid. 60, 708 (1999).

[10] C. Monroe, D. M. Meekhof, B. E. King, and D. J. Wineland, Science 272, 1131 (1996). 